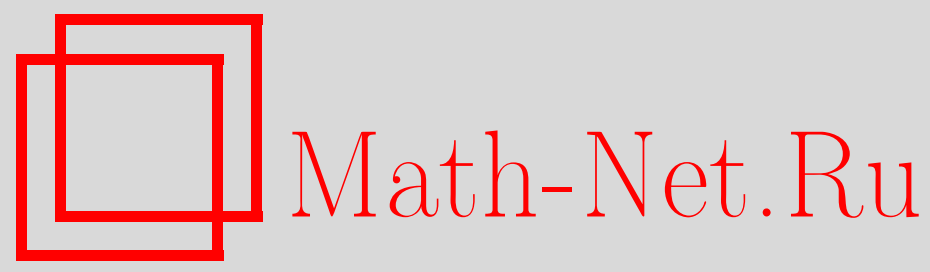

Е. А. Иванова, Об аппроксимируемости относительно сопряженности конечными $p$-группами свободных произведений двух групп с объединенной подгруппой, Матем. заметки, 2004, том 76, выпуск 4, 502-509

DOI: https://doi.org/10.4213/mzm126

Использование Общероссийского математического портала Math-Net.Ru подразумевает, что вы прочитали и согласны с пользовательским соглашением http://www . mathnet.ru/rus/agreement

Параметры загрузки:

IP : 54.92 .164 .108

26 апреля 2023 г., 14:22:14

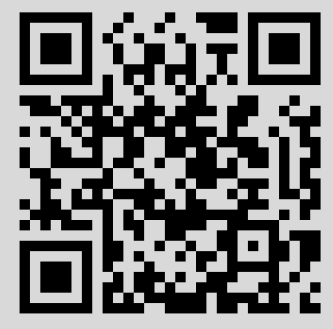




\title{
ОБ АППРОКСИМИРУЕМОСТИ ОТНОСИТЕЛЬНО СОПРЯЖЕННОСТИ КОНЕЧНЫМИ $p$-ГРУППАМИ СВОБОДНЫХ ПРОИЗВЕДЕНИЙ ДВУХ ГРУПП С ОБЪЕДИНЕННОЙ ПОДГРУППОЙ
}

\section{Е. А. Иванова}

\begin{abstract}
Доказано, что свободное произведение двух конечных $p$-групп с объединенными центральньпи подгруппами является группой, аппроксимируемой относительно сопряженности конечными $p$-группами. С использованием этого результата показано, что для свободного произведения с объединенньми подгруппами двух конечно порожденных абелевых групп свойства аппроксимируемости конечньми $p$-группами относительно отношения равенства и относительно отношения сопряженности являются равносильными.

Библиограффия: 8 названий.
\end{abstract}

1. Введение. Напомним, что группа $G$ называется финитно аппроксимируемой (соответственно аппроксимируемой конечными р-группами) относительно сопряженности, если для любых элементов $a$ и $b$ этой групы, не сопряженных в ней, найдется гомоморфизм группы $G$ на конечную группу (соответственно на конечную $p$-групш) $X$, образы относительно которого элементов $a$ и $b$ не сопряжены в $X$.

Напомним также, что подмножество $M$ группы $G$ называется финитно отделимым (соответственно отделимымм в классе конечных $p$-групп), если для любого элемента $a \in G$, не принадлежашего подмножеству $M$, существует гомоморфизм $\varphi$ группы $G$ на конечную групу (соответственно конечную $p$-группу) $X$ такой, что $a \varphi \notin M \varphi$.

Элемент $a$ группы $G$ будем называть $C_{f}$-отделимым (соответственно $C_{f p}$-отделимым.м, если множество $a^{G}=\left\{x^{-1} a x \mid x \in G\right\}$ элементов, сопряженных с $a$ в групше $G$, является финитно отделимым (соответственно отделимым в классе конечных $p$-групा).

Таким образом, группа $G$ является финитно аппроксимируемой (соответственно аппроксимируемой конечными $p$-группами) относительно сопряженности тогда и только тогда, когда каждьй элемент этой групшы $C_{f}$-отделим (соответственно $C_{f p}$-отделим).

Очевидно, что если некоторая група является финитно аппроксимируемой относительно сопряженности, то она финитно аппроксимируема (т.е. аппроксимируема конечными группами относительно равенства). Столь же очевидно аналогичное утверждение для аппроксимируемости конечными $p$-группами. Несмотря на то, что соответствующие обратные утверждения, вообще говоря, не имеют места, в ряде случаев оказывается, что условия, гарантируюшие финитную аппроксимируемость групп некоторого класса, обеспечивают и финитную аппроксимируемость их относительно сопряженности. Так, в работе Дайер [1] доказано, что свободное произведение с объединенной 
подгруппой двух групп является группой, финитно апшроксимируемой относительно сопряженности, если свободные множители являются конечньпи группами (финитная аппроксимируемость таких груп была установлена Баумслагом [2] еще в 1963 году).

Свободное произведение с объединенной подгруппой двух конечных $p$-групा не обязательно является группой, аппроксимируемой конечньми $p$-группами; условия, необходимые и достаточные для этого, указаны в работе Хигмена [3]. Возникает естественный вопрос, будут ли эти условия обеспечивать и апшроксимируемость таких свободных произведений конечньми $p$-группами относительно сопряженности? В этом направлении здесь будет доказана

Теорема 1. Пусть $A$ и $B$ - конечные $p$-групnы, $H$ - nодгруппа групnы $A, K$ подәруппа группы $B u \varphi: H \rightarrow K-$ изоморфизм группы $H$ на группу $K$. Пусть $G=(A * B ; H=K, \varphi)-$ свободное произведение групп $A$ и $B$ с обтединенными относительно $\varphi$ подгруппами $H$ и $K$. Тогда

(1) если әруппа $G$ аппроксимируема конечными р-группами, то все ее әлементы бесконечного порядка являются $C_{f р}$-отделимыми;

(2) если $H$ и $K$ являются чентральными подгруппами групп $A$ и $B$ соответственно, то группа $G$ аппроксимируема конечными р-группами относительно сопряхенности.

Заметим, что апшроксимируемость групшы $G$ конечными $p$-группами при условии центральности подгруп $H$ и $K$ вытекает непосредственно из упомянутого вьше критерия Хигмена.

С помошью теоремы 1 можно показать, что в случае, когда групшы $A$ и $B$ являются абелевыми с конечным числом порождающих, аппроксимируемость групшы $G$ конечными $p$-группами относительно равенства и относительно сопряженности являются равносильньми свойствами. Более точно, имеет место

Теорема 2. Пусть $G=(A * B ; H=K, \varphi)$ - свободное произведение с обтединенными подгруппами конечно порожденных абелевых групп $A$ и $B$, аппроксимируемых конечными р-группами, причем $H \neq A$ и $K \neq B$. Следующие утвержсдения равносильны:

(1) группа $G$ аппроксимируема конечными р-группами относительно сопряжсенности;

(2) группа $G$ аппроксимируема конечными р-группами;

(3) подгруппы $H$ и $K p^{\prime}$-изолированы в группах $A$ и $B$ соответственно.

Напомним, что если $p$ - простое число, то подгруппа $X$ некоторой групшы $Y$ называется $p$-изолированной, если для любого элемента $y \in Y$ из включения $y^{p} \in X$ следует, что $y \in X$. Подгруппа $X$ группы $Y$ назьвается $p^{\prime}$-изолированной, если она $q$-изолирована для любого простого числа $q \neq p$.

В работе [4] показано, что если свободное произведение $G=(A * B ; H=K, \varphi)$ локально нильпотентных групп $A$ и $B$, где $H \neq A$ и $K \neq B$, является группой, аппроксимируемой нильпотентными группами, то для некоторого простого числа $p$ подгрупшы $H$ и $K p^{\prime}$-изолированы в группах $A$ и $B$ соответственно. Поэтому имеет место

СлЕДСТВИЕ 3. Пусть $G=(A * B ; H=K, \varphi)$ - свободное произведение с оббединенными подгруппами конечно порожденных свободных абелевых групп $A$ и $B$, 
причем $H \neq A$ и $K \neq B$. Следуюшие утвер сдения равносильны:

(1) группа $G$ аппроксимируема нильпотентными группами;

(2) для некоторого простого числа р подгруппь $H$ и $K p^{\prime}$-изолированы в группах $A$ и В соответственно;

(3) для некоторого простого числа р группа $G$ аппроксимируема конечными р-группами относительно сопрязсенности;

(4) для некоторого простого числа р группа $G$ аппроксимируема конечными р-группами.

2. Доказательство теоремы 1. Начнем с доказательства двух вспомогательных утверждений, являющихся аналогами соответствующих результатов для $C_{f}$-отделимости, полученных в работе [5] (лемма 1 и теорема 1 соответственно).

ПРЕДЛОЖЕНИЕ 4. Пусть $H$ - субнормальная подгруппа конечного р-индекса группы $G$. Если әлемент $h \in H$ является $C_{f p}$-отделимым в группе $H$, то он является $C_{f p}$-отделимым и в группе $G$.

ДокАЗАТЕЛЬСТво. Очевидная индукция по длине последовательности подгрупп

$$
H=H_{0} \leqslant H_{1} \leqslant \cdots \leqslant H_{s}=G,
$$

где для $i=0,1, \ldots, s-1$ подгруппа $H_{i+1}$ совпадает с нормализатором в группе $G$ подгруппы $H_{i}$, позволяет считать, что $H$ является нормальной подгруппй групшы $G$.

Предположим, что элемент $h \in H$ является $C_{f p}$-отделимым в группе $H$, и пусть $g-$ произвольный элемент групшы $G$, не сопряженньй с элементом $h$. Покажем, что для подходящей нормальной подгрупшы $N$ конечного $p$-индекса группы $G$ в фактор-группе $G / N$ элементы $h N$ и $g N$ не являются сопряженными.

Если элемент $g$ не принадлежит подгруппе $H$, то подгруппа $N=H$ является, очевидно, искомой. Пусть $g \in H$. Фиксируем систему $c_{1}, c_{2}, \ldots, c_{n}$ представителей левых смежных классов группы $G$ по подгрупше $H$ и полагаем для $i=1,2, \ldots, n g_{i}=c_{i}^{-1} g c_{i}$. Так как каждый из элементов $g_{1}, g_{2}, \ldots, g_{n}$ групшы $H$ не сопряжен в этой группе с элементом $h$, по предположению найдется такая нормальная подгрупша $M$ конечного $p$-индекса группы $H$, что в фактор-группе $H / M$ образ каждого из элементов $g_{1}, g_{2}, \ldots, g_{n}$ не сопряжен с образом элемента $h$.

Пусть $M_{i}=c_{i}^{-1} M c_{i}, i=1,2, \ldots, n$. Так как сопряжение произвольным элементом группы $G$ индуцирует некоторьй автоморфизм группы $H$, каждая из подгрупп $M_{1}, M_{2}$, $\ldots, M_{n}$ инвариантна в $H$ и имеет в этой группе конечный $p$-индекс. Поэтому их пересечение $N$ является подгруппой конечного $p$-индекса групшы $H$, а потому и групшы $G$. Очевидно, кроме того, что подгруппа $N$ инвариантна в группе $G$ и что в фактор-групе $G / N$ образ элемента $g$ не сопряжен с образом элемента $h$.

ПРЕДЛОЖЕНИЕ 5. Если группа $G$ является расиирением свободной группы при помощи конечной p-группы, то в группе $G$ каждый әлемент бесконечного порядка $C_{f p}$-отделим.

ДокАЗАТЕЛЬСТво. Пусть $F$ - свободная нормальная подгруппа конечного $p$-индекса группы $G$ и $a \in G$ - элемент бесконечного порядка. Если $H$ - подгруппа группы $G$, порожденная подгруппй $F$ и элементом $a$, то $H$ имеет конечньй $p$-индекс в групе $G$ и 
является субнормальной, поскольку фактор-группа $G / F$ нильпотентна. Поэтому ввиду предложения 4 можно без потери общности считать, что $G=H$. Это означает, в частности, что произвольньй элемент $g \in G$ записывается в виде $g=a^{k} f$ для некоторого целого числа $k$ и подходящего элемента $f \in F$.

Пусть $b$ - произвольный элемент групшы $G$, не сопряженный с элементом $a$. Если элементы $a$ и $b$ принадлежат разным смежным классам по подгрупше $F$, то поскольку фактор-группа $G / F$ абелева, элементы $a F$ и $b F$ не сопряжены в конечной $p$-групше $G / F$.

Будем считать теперь, что $a F=b F$. Поскольку фактор-группа $G / F$ является конечной $p$-группой, то для некоторого целого числа $n \geqslant 0$ должно выполняться включение $a^{p^{n}} \in F$. Докажем, что элементы $a^{p^{n}}$ и $b^{p^{n}}$ не сопряжены в групе $G$.

Пусть, напротив, для некоторого элемента $g \in G$ имеет место равенство $b^{p^{n}}=$ $g^{-1} a^{p^{n}} g$. Записьвая элемент $g$ в виде $g=a^{k} f$, где $f \in F$, имеем $b^{p^{n}}=f^{-1} a^{p^{n}} f$. Пусть $a_{1}=f^{-1} a f$. Тогда $a_{1} F=a F$, и потому $b=a_{1} x$ для подходящего $x \in F$. Из равенства $\left(a_{1} x\right)^{p^{n}}=a_{1}^{p^{n}}$ следует, очевидно, перестановочность элементов $a_{1} x$ и $a_{1}^{p^{n}}$, откуда, в свою очередь, вытекает, что элементы $x$ и $a_{1}^{p^{n}}$ свободной группы $F$ перестановочны. Следовательно, эти элементы должны принадлежать некоторой циклической подгруппе групшы $F$, т.е. для некоторого элемента $y \in F$ и подходящих целых чисел $r$ и $s$ выполнены равенства $x=y^{r}$ и $a_{1}^{p^{n}}=y^{s}$. При этом, ввиду того, что порядок элемента $a$ бесконечен, $s \neq 0$. В силу второго из этих равенств элементы $a_{1}$ и $y^{s}$ являются перестановочньми, т.е. $\left(a_{1}^{-1} y a_{1}\right)^{s}=y^{s}$. Так как $a_{1}^{-1} y a_{1} \in F$ и в свободной групше извлечение корней однозначно, отсюда следует перестановочность элементов $a_{1}$ и $y$. Поэтому из равенства $\left(a_{1} x\right)^{p^{n}}=a_{1}^{p^{n}}$ следует, что $x^{p^{n}}=1$, т.е. $x=1$. Отсюда $b=a_{1}$, что невозможно, так как элементы $a$ и $b$ не являются сопряженньми.

Итак, элемент $b^{p^{n}}$ не сопряжен в групше $G$ с элементом $a^{p^{n}}$ из подгрупшы $F$. Так как свободная группа аппроксимируема конечньми $p$-группами относительно сопряженности для любого простого числа $p$ (см. [6, предложение 4.8]), из предложения 4 следует существование такой нормальной подгруппы $N$ конечного $p$-индекса группы $G$, что в фактор-группе $G / N$ образы элементов $a^{p^{n}}$ и $b^{p^{n}}$ не сопряжены. Очевидно, что тогда и образы элементов $a$ и $b$ не являются сопряженными, и предложение 5 доказано.

Перейдем теперь непосредственно к доказательству теоремы 1. Хорошо известно (и легко показать, используя теорему Нейман (см., например, [7, следствие 4.9.2]), что свободное произведение с объединенными подгруппами $G=(A * B ; H=K, \varphi)$ двух конечных $p$-групп $A$ и $B$ является группой, аппроксимируемой конечньми $p$-группами, тогда и только тогда, когда $G$ есть расширение свободной группы при помощи конечной $p$-группы. Поэтому первое утверждение теоремы 1 сразу же следует из предложения 5 .

Как упоминалось во введении, из теоремы Хигмена [3] следует, что если объединяемые подгрупшы $H$ и $K$ являются центральными в группах $A$ и $B$ соответственно, то группа $G$ апшроксимируема конечными $p$-грушами. Поэтому ввиду того же предложения 5 для доказательства второго утверждения теоремы 1 достаточно рассматривать лишь те несопряженные элементы грушшы $G$, порядки которых конечны.

Итак, пусть $x$ и $y$ - элементы конечного порядка группы $G$, не сопряженные в этой группе. Поскольку каждьй элемент конечного порядка свободного произведения груш с объединенной подгруппой сопряжен с элементом одного из свободных множителей, без потери общности можно считать, что каждьй из элементов $x$ и $y$ лежит в одной из подгрупп $A$ и $B$. Если, при этом, эти элементы принадлежат одной и той же подгруппе $A$ 
или $B$, то они не сопряжены в этой подгруппе.

Поскольку подгрупшы $H$ и $K$ являются центральными в группах $A$ и $B$, можно построить обобщенное прямое произведение $P$ групп $A$ и $B$ с подгруппами $H$ и $K$, объединенными относительно изоморфизма $\varphi$. Напомним (см., например, [8]), что группа $P$ может быть определена как фактор-группа прямого произведения $A \times B$ групा $A$ и $B$ по подгруппе $N$, состоящей из всевозможных элементов вида $h(h \varphi)^{-1}$, где $h \in H$.

Без труда проверяется, что произведение $\alpha$ стандартного вложения групшы $A$ в группу $A \times B$ и естественного гомоморфизма групшы $A \times B$ на групш $P$ является вложением группы $A$ в групш $P$; аналогично определяется вложение $\beta$ групшы $B$ в группу $P$. Кроме того, $A \alpha \cap B \beta=H \alpha=K \beta$, произвольный элемент из подгруппы $A \alpha$, не принадлежащий подгруппе $H \alpha$, не сопряжен в группе $P$ ни с одним элементом из подгруппы $B \beta$, и два элемента из подгруппы $A \alpha$ (или из подгруппы $B \beta$ ) сопряжены в групе $P$ тогда и только тогда, когда они сопряжены в подгруппе $A \alpha$ (соответственно в подгрупше $B \beta$ ).

Так как для любого элемента $h \in H$ вьполнено равенство $h \alpha=h(\varphi \beta)$, то существует гомоморфизм $\rho$ групшы $G$ в группу $P$, продолжающий отображения $\alpha$ и $\beta$. Очевидно теперь, что элементы $x \rho$ и $y \rho$ не сопряжены в конечной $p$-групе $P$, и доказательство теоремы 1 закончено.

3. Доказательство теоремы 2. Импликация $(1) \Longrightarrow(2)$ в теореме 2 очевидна. Легко устанавливается и справедливость импликации $(2) \Longrightarrow(3)$.

В самом деле, пусть групша $G=(A * B ; H=K, \varphi)$ апшроксимируема конечными $p$-групшами, причем групшы $A$ и $B$ абелевы и $H \neq A, K \neq B$. Если, скажем, подгруппа $H$ не является $p^{\prime}$-изолированной в групше $A$, то для некоторого элемента $a \in A \backslash H$ и некоторого простого числа $q \neq p$ имеет место включение $a^{q} \in H$. Пусть еще $b$ - произвольный элемент группы $B$, не принадлежащий подгрупе $K$. Тогда запись $[a, b]=a^{-1} b^{-1} a b$ коммутатора элементов $a$ и $b$ несократима, и потому этот коммутатор является неединичным элементом группы $G$. С другой стороны, легко видеть, что для любого гомоморфизма $\theta$ группы $G$ на конечную $p$-группу выполнено включение $a \theta \in H \theta$, так что оба элемента $a \theta$ и $b \theta$ принадлежат абелевой группе $B \theta$. Таким образом, элемент $[a, b]$ переходит в 1 при любом гомоморфизме группы $G$ на конечную $p$-группу, что противоречит предположению об аппроксимируемости группы $G$ конечными $p$-группами.

Таким образом, остается доказать, что из $p^{\prime}$-изолированности в группах $A$ и $B$ подгрупп $H$ и $K$ следует аппроксимируемость групшы $G$ конечными $p$-группами относительно сопряженности.

Для этого необходимо, прежде всего, напомнить следующее понятие (см. [2]). Если $A$ и $B$ - некоторые групшы, $H$ - подгруппа группы $A, K$ - подгруппа групшы $B$ и $\varphi: H \rightarrow K$ - изоморфизм, то подгруппы $R \leqslant A$ и $S \leqslant B$ назьваются $(H, K, \varphi)$-совместимыми, если $(H \cap R) \varphi=K \cap S$. Если нормальные подгруппы $R$ и $S$ групп $A$ и $B$ соответственно $(H, K, \varphi)$-совместимы, то отображение $\varphi_{R, S}: H R / R \rightarrow K S / S$, определяемое по правилу

$$
(h R) \varphi_{R, S}=(h \varphi) S, \quad h \in H,
$$

является изоморфизмом подгрупшы $H R / R$ фактор-группы $A / R$ на подгруппу $K S / S$ фактор-группы $B / S$. Поэтому можно построить свободное произведение

$$
G_{R, S}=\left(A / R * B / S ; H R / R=K S / S, \varphi_{R, S}\right)
$$


групп $A / R$ и $B / S$ с подгруппами $H R / R$ и $K S / S$, объединенньми в соответствии с изоморфизмом $\varphi_{R, S}$. Естественные отображения группы $A$ на фактор-группу $A / R$ и группы $B$ на фактор-групшу $B / S$ продолжаемы до гомоморфизма $\rho_{R, S}$ групшы $G=(A * B$; $H=K, \varphi)$ на группу $G_{R, S}$.

Если группы $A$ и $B$ абелевы и индексы $(H, K, \varphi)$-совместимьх подгрупп $R$ и $S$ в группах $A$ и $B$ конечны и являются $p$-числами, то в силу теоремы 1 группа $G_{R, S}$ аппроксимируема конечными $p$-группами относительно сопряженности. Поэтому для доказательства апшроксимируемости относительно сопряженности конечньпи $p$-групами группы $G$ достаточно показать, что для любых несопряженньх элементов $x$ и $y$ группы $G$ можно найти такие $(H, K, \varphi)$-совместимые подгрупшы $R$ и $S$ конечных $p$-индексов в группах $A$ и $B$, что образы $x \rho_{R, S}$ и $y \rho_{R, S}$ этих элементов не сопряжены в группе $G_{R, S}$.

Напомним еще, что произвольный элемент $x$ группы $G=(A * B ; H=K, \varphi)$ может быть записан в виде $x=x_{1} x_{2} \ldots x_{n}$, где каждый из сомножителей $x_{1}, x_{2}, \ldots, x_{n}$ принадлежит одной из подгрупп $A$ или $B$ и при $n>1$ любые соседние сомножители $x_{i}$ и $x_{i+1}$ не лежат в одной и той же из этих подгрупп (и потому не входят в соответствующую объединяемую подгруппу $H$ или $K$ ). Такая запись элемента $x$ называется его несократимой записью, а число $n$ сомножителей этой записи (одно и то же для всех несократимых записей данного элемента) называется длиной элемента $x$. Элемент $x$ групы $G$ называется ииклически несократимым, если либо его длина $n$ равна 1 , либо $n>1$ и сомножители $x_{1}$ и $x_{n}$ его несократимой записи не принадлежат одной и той же подгруппе $A$ или $B$. В этом случае для каждого $i=1,2, \ldots, n$ запись $u_{i}=x_{i} x_{i+1} \ldots x_{n} x_{1} \ldots x_{i-1}$ элемента $u_{i}$ является несократимой; элементы $u_{1}, u_{2}, \ldots, u_{n}$ назьваются ииклическими перестановками элемента $x$ (отметим, что при $n=1$ единственной циклической перестановкой элемента $x$ является он сам).

Общий критерий Солитэра сопряженности двух элементов свободного произведения групп с объединенной подгруппой (теорема 4.6 из [7]) в рассматриваемом здесь случае абелевых свободных множителей принимает более простой вид.

ПРЕДЛОЖЕНИЕ 6. Пусть $G=(A * B ; H=K, \varphi)$ - свободное произведение с оббединенными подгруппами абелевых групп $A$ и $B$. Произвольный әлемент группы $G$ сопряжен с некоторым ииклически несократимым әлементом. Циклически несократимые әлементы $x$ и у сопряжены в группе $G$ тогда и только тогда, когда их длины равны и один из них совпадает с некоторой ииклической перестановкой другого.

Для построения искомых гомоморфизмов $\rho_{R, S}$ необходимо доказать два предварительных утверждения.

ПрЕдЛОЖЕНИЕ 7. Пусть $A$ и В - конечно порожденные абелевы группы, $H$ $p^{\prime}$-изолированная подгруппа группы $A, K-p^{\prime}$-изолированная подгруппа группь $B u$ $\varphi: H \rightarrow K-$ изоморфизм. Для любых подгрупп $M$ u $N$ конечных $p$-индексов групп $A$ $u$ B соответственно существуют $(H, K, \varphi)$-совместимые подгруппь $R \leqslant A u$ $S \leqslant B$, имеющие конечные р-индексы в группах $A$ и $B$ и такие, что $R \leqslant M u$ $S \leqslant N$.

ДокАЗАТЕЛьСтво. Докажем сначала следующий вспомогательньй результат.

Лемма 8. Пусть $A$ - конечно порожденная абелева группа, $H-p^{\prime}$-изолированная подгруппа группь $A$ и $U$ - подгруппа конечного р-индекса группы $Н$. Для любой 
подгруппы $M$ конечного р-индекса группы $A$, содержащей подгруппу $U$, существует подгруппа $R$ конечного р-индекса группы $A$ такая, что $R \leqslant M u R \cap H=U$.

В самом деле, так как подгруппа $U$ является, очевидно, $p^{\prime}$-изолированной в группе $A$, фактор-группа $A / U$ оказьвается конечно порожденной абелевой группой без $p^{\prime}$-кручения и потому аппроксимируема конечными $p$-группами. Поскольку ее подгруппа $H / U$ конечна, найдется подгруппа $R / U$ конечного $p$-индекса группы $A / U$, не имеющая с подгруппой $H / U$ общих неединичных элементов. Так как, при этом, можно считать без потери общности, что $R / U$ содержится в подгруппе $M / U$, подгруппа $R$ является искомой.

Пусть теперь $M$ и $N$ - подгруппы конечных $p$-индексов групп $A$ и $B$ соответственно. Тогда $U=(M \cap H) \cap(N \cap K) \varphi^{-1}$ - подгрупша конечного $p$-индекса группы $H$ и $V=(M \cap H) \varphi \cap(N \cap K)$ - подгруппа конечного $p$-индекса группы $K$. В соответствии с леммой 8 выберем в группе $A$ такую подгрупшу $R$ конечного $p$-индекса, что $R \leqslant M$ и $R \cap H=U$, а в групе $B$ - такую подгрупшу $S$ конечного $p$-индекса, что $S \leqslant N$ и $S \cap K=V$. Так как $U \varphi=V$, подгрупшы $R$ и $S$ являются искомыми. Предложение 7 доказано.

ПРЕДЛОЖЕНИЕ 9. Пусть $A$ и $B$ - конечно порожденные абелевы группь, аппроксимируемые конечными р-группами, $H$ - $p^{\prime}$-изолированная подгруппа группы $A, K-p^{\prime}$-изолированная подгруппа группы $B u \varphi: H \rightarrow K-$ изоморфизм. Для любого конечного набора $g_{1}, g_{2}, \ldots, g_{r}$ неединичных әлементов группь $G=(A * B$; $H=K, \varphi)$ найдутся $(H, K, \varphi)$-совместимые подгруппы $R \leqslant A u S \leqslant B$ конечных $p$-индексов такие, что для любого $i=1,2, \ldots, r$ образ $g_{i} \rho_{R, S}$ әлемента $g_{i}$ является неединичным әлементом группы $G_{R, S}$. Более того, подгруппь $R$ и $S$ мохно выбрать так, что для каждого $i=1,2, \ldots, r$ длина әлемента $g_{i} \rho_{R, S}$ в группе $G_{R, S}$ совпадает с длиной әлемента $g_{i}$ в группе $G$ ( $и$ потому әлемент $g_{i} \rho_{R, S}$ является ииклически несократимым, если әлемент $g_{i}$ ииклически несократим).

ДокАЗАТЕльство. Это утверждение является непосредственным следствием предложения 7. Действительно, если длина элемента $g_{i}$ больше 1 , то все сомножители его несократимой записи лежат попеременно в группах $A$ и $B$ и не входят в соответствующие объединяемые подгруппы $H$ и $K$. Поскольку фактор-групшы $A / H$ и $B / K$ аппроксимируемы конечными $p$-группами, найдутся такие подгруппы $M_{i}$ и $N_{i}$ конечных $p$-индексов групп $A$ и $B$ соответственно, что все сомножители несократимой записи элемента $g_{i}$, лежащие в групе $A$, не входят в подгруппу $M_{i} H$, а сомножители, лежашие в группе $B$, не входят в подгруппу $N_{i} K$. Если же длина элемента $g_{i}$ равна 1 , то, воспользовавшись предположением об апшроксимируемости групा $A$ и $B$ конечными $p$-группами, подгруппы $M_{i} \leqslant A$ и $N_{i} \leqslant B$ конечных $p$-индексов выберем так, чтобы элемент $g_{i}$ не входил в подгруппу $M_{i}$, если $g_{i} \in A$, и не входил в подгруппу $N_{i}$, если $g_{i} \in B$. Полагая

$$
M=\bigcap_{i=1}^{r} M_{i} \text { и } N=\bigcap_{i=1}^{r} N_{i},
$$

видим, что $(H, K, \varphi)$-совместимые подгрупшы $R$ и $S$, выбранные в соответствии с предложением 7 так, чтобы $R \leqslant M$ и $S \leqslant N$, являются искомыми.

Пусть $G=(A * B ; H=K, \varphi)$ - свободное произведение с объединенными подгруппами конечно порожденных абелевых групп $A$ и $B$, аппроксимируемых конечными 
$p$-группами, причем объединяемые подгрупшы $H$ и $K$ являются $p^{\prime}$-изолированными в группах $A$ и $B$ соответственно. Пусть циклически несократимые элементы $x$ и $y$ группы $G$ не сопряжены в этой группе. Напомним, что для завершения доказательства теоремы 2 нам достаточно указать такие $(H, K, \varphi)$-совместимые подгруппы $R$ и $S$ конечных $p$-индексов в группах $A$ и $B$, что образы $x \rho_{R, S}$ и $y \rho_{R, S}$ этих элементов не сопряжены в группе $G_{R, S}$.

Если длины этих элементов различны, то в соответствии с предложением 9 найдутся $(H, K, \varphi)$-совместимые подгрупшы $R \leqslant A$ и $S \leqslant B$ конечных $p$-индексов такие, что образы $x \rho_{R, S}$ и $y \rho_{R, S}$ этих элементов также циклически несократимы и их длины различны. Так как тогда в силу предложения 6 элементы $x \rho_{R, S}$ и $y \rho_{R, S}$ не сопряжены в группе $G_{R, S}$, подгруппы $R$ и $S$ искомые.

Пусть теперь длина каждого из элементов $x$ и $y$ равна $n$, и пусть $y_{1}, y_{2}, \ldots, y_{n}-$ все циклические перестановки элемента $y$. Поскольку элементы $x$ и $y$ не сопряжены в группе $G$, элемент $x$ отличен от каждого из элементов $y_{1}, y_{2}, \ldots, y_{n}$. Из предложения 9 вытекает существование $(H, K, \varphi)$-совместимых подгрупा $R \leqslant A$ и $S \leqslant B$ конечных $p$-индексов таких, что образы относительно гомоморфизма $\rho_{R, S}$ элементов $x$ и $y$ имеют длину $n$, циклически несократимы и образ элемента $x$ отличен от образа каждого из элементов $y_{1}, y_{2}, \ldots, y_{n}$. Легко видеть, кроме того, что произвольная циклическая перестановка элемента $y \rho_{R, S}$ является образом одного из элементов $y_{1}, y_{2}, \ldots, y_{n}$. Из предложения 6 теперь следует, что элементы $x \rho_{R, S}$ и $y \rho_{R, S}$ не сопряжены в групе $G_{R, S}$. Теорема 2 доказана.

\section{СПИСОК ЦИТИРОВАННОЙ ЛИТЕРАТУРЫ}

[1] Dyer J. L. Separating conjugates in amalgamated free products and HNN extensions // Austral. Math. Soc. Ser. A. 1980. V. 29. P. 35-51.

[2] Baumslag G. On the residual finiteness of generalised free products of nilpotent groups // Trans. Amer. Math. Soc. 1963. V. 106. P. 193-209.

[3] Higman G. Amalgams of $p$-groups // J. Algebra. 1964. V. 1. P. 301-305.

[4] Азаров Д. Н., Иванова Е. А. О нильпотентной аппроксимируемости свободного произведения с объединением локально нильпотентных групп // Науч. тр. ИвГУ. Сер. Матем. Т. 2. Иваново: Изд-во ИвГУ, 1999. С. 5-7.

[5] Stebe P.F. A residual property of certain groups // Proc. Amer. Math. Soc. 1970. V. 26. P. 37-42.

[6] Линдон Р., Шупп П. Комбинаторная теория групп. М.: Мир, 1980.

[7] Магнус В., Каррас А., Солитэр Д. Комбинаторная теория групп. М.: Наука, 1974.

[8] Neumann B. H. An essay on free products of groups with amalgamations // Philos. Trans. Roy. Soc. London Ser. A. 1954. V. 246. P. 503-554. 